\title{
Study on Application of Virtual Reality Technique in Environmental Artistic \\ Design
}

Wu Jing

Art College of ShanDong University (WeiHai) 264200

\begin{abstract}
With the advent of the information age, people's lives have been changed unknowingly owing to lots of modern technology means. For the environmental artistic design, the traditional mode of environmental artistic design is broken through after the emergence of computer technology and virtual reality technique, which makes the scheme and application of virtual reality technique in environmental artistic design to own more practical value. In this paper, the virtual reality technique and its practical application in environmental artistic design are set forth, and the profound influence on the actual design by the virtual reality technique is studied with expectation of enlightenment for broadening the thought of environmental artistic design.
\end{abstract}

Keywords: Virtual reality technique, Environmental artistic design, Application study

\section{INTRODUCTION}

At the present stage, the rapid growth of information technology and the advent of kinds of multimedia technology have driven the new development of practical work. With the increasingly intense competition in the market, how to improve modern environmental artistic design becomes the common topic concerned by the experts and scholars in this field. In the past, the environmental artistic design alternative often can't be promoted due to funds, space and other factors. Thus, consolidation of the virtual reality technique and the environmental artistic design is put forward in order to strengthen efficiency and economy of the environmental artistic design. In the process of actual design in the practical application of virtual reality technique, the shortcomings of traditional environmental artistic design can be made up for by multi-perceptibility, reciprocity, concept and other technical features of virtual reality technique.

\section{Overview of Virtual Reality Technique} (1) Analysis of Virtual Reality Technique.

The virtual reality technique essentially is a serial of advanced technology, including multimedia technology, computer network technology, simulation technique and other techniques, which all creates a kind of realistic three-dimensional virtual environment. Under the support of hardware equipment, the creation of multi-dimensional information space is realized, and then the intuitive and three-dimensional virtual environment is shown in the specific field ${ }^{[1]}$. 


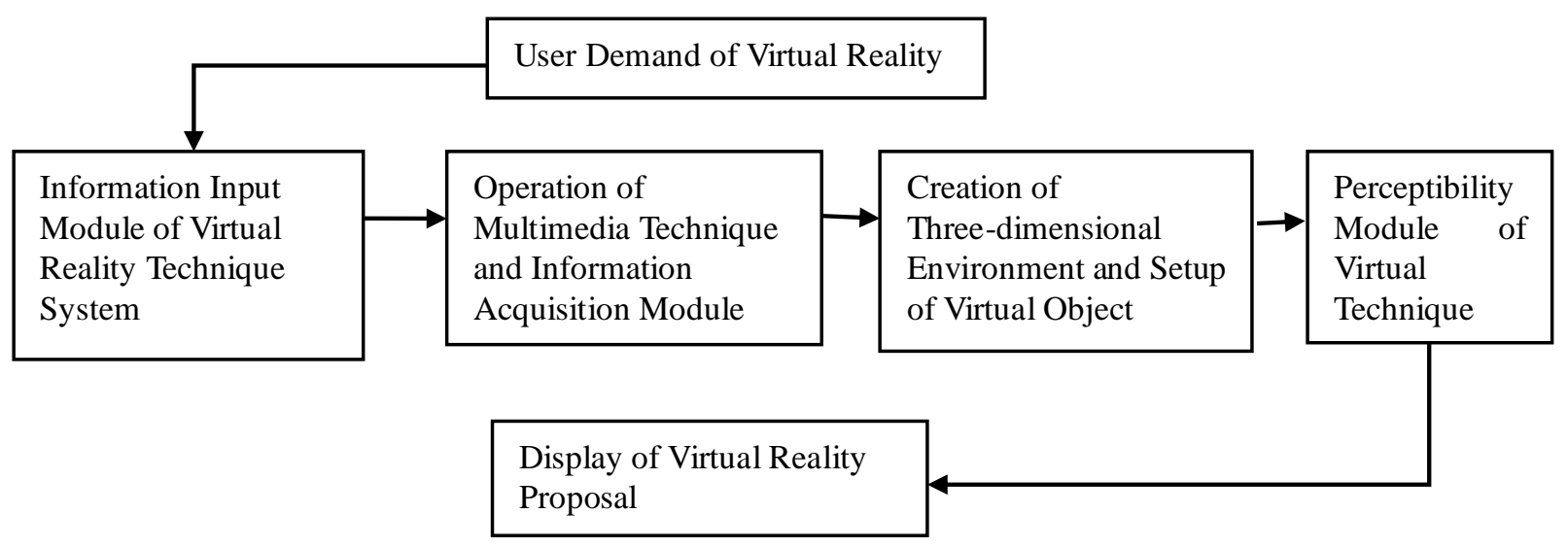

Fig. 1 Flow Diagram of Implementation Procedure of Virtual Reality Technique Proposal

From Fig. 1, we can clearly observe that the virtual reality technique is implemented under the instruction of requirements specified by the users and different virtual scene shall be realized on the basis of different techniques. On the whole, most of virtual reality technique should be applied based on the setup of information input module of virtual reality system, and the foundation of virtual environment can be created by the specific operation of multimedia technology and information acquisition module, in order to build three-dimensional space of environment and furnish the concrete virtual objects on the basis of the foundation of virtual environment to make it more realistic. Moreover, the most important is that the interactions between people and the environment are generated in the actual application process of virtual reality technique when the operation of perceptibility module of virtual technique, and then the virtual reality proposals are presented to the audiences by multimedia technology, computer information technology and other means.

\section{(2) Analysis of Main Features of Virtual}

Reality Technique. The creation of virtual reality circumstance is to build a simulation environment based on the computer technology as the core and generate a realistic virtual environment relying on the modern high-tech hardware and software products, in order to create an intuitive three-dimensional scene and adopt a series of measures to stimulate the visual sense, auditory sense, touch sense and other sensory feelings of audiences on site, to make people being in like a real and actual environment $^{[2]}$. Specifically, there are main features for the virtual reality technique as follows:

\section{Multi-Perceptibility of Virtual Reality} Technique. Usually, we have the perception of the objects through our visual, touch or auditory sense. While the operation of virtual reality technique, we hope to have the most ideal outcome that all the sensing capability of people can be aroused for the purpose of panoramic observation or overall consideration ${ }^{[3]}$. However, this idealized virtual reality technique can't reach the peak under current technology, which needs to further develop its technical feature of 
multi-perceptibility in the future.

\section{Reciprocity of Virtual Reality Technique.} The reciprocity of virtual reality technique is mainly reflected in its interactive effects. Namely, people can interact with the virtual scene and objects in the environment of virtual technique. Although this interaction is the concretization of perceptive scene, the practical value of the presented technique is very high, and then the possible results arisen in the real environment can be estimated.

\section{Conception of Virtual Reality Technique.}

In simple terms, the conception of virtual reality technique refers to the image of the virtual environment to meet the demands of people on the exploration of objectively non-existing scene. Although the conception feature of the technique is obvious, the imaged scene often comes from people's cognition of things and imagination of unknown world. Thus, the conception in the virtual reality technique is achieved in the operation of multiple supplementary techniques.

\section{Study on Practical Application of Virtual Reality Technique in Environmental Artistic Design}

The status of environmental artistic design can be visually presented by the virtual reality technique, which not only contributes to strengthen the budgetary accuracy of environmental artistic design and make the bilateral interaction unceasingly enhancing in the process of design, but also favors to comprehensively display the environmental artistic design proposal and its background, change the condition of traditional environmental artistic design due to the limitation of thought expression and make the environmental artistic design to own more dynamic feature ${ }^{[4]}$.

\section{(1) Analysis on Purpose and Intrinsic Value} of Environmental Artistic Design. On the whole, the intrinsic value in fields of architectural design and environmental design in China shall be presented by all types of advanced techniques. Of which, the purpose of environmental artistic design is mainly to display the valuable design alternative with the most economical cost. In practice, it is necessary to take all the feasible means to improve the quality and efficiency and break through the development bottleneck of environmental artistic design. The unique connotation of environmental artistic design can be observed from the design chart of virtual scene built, specifically shown in Fig. 1: 


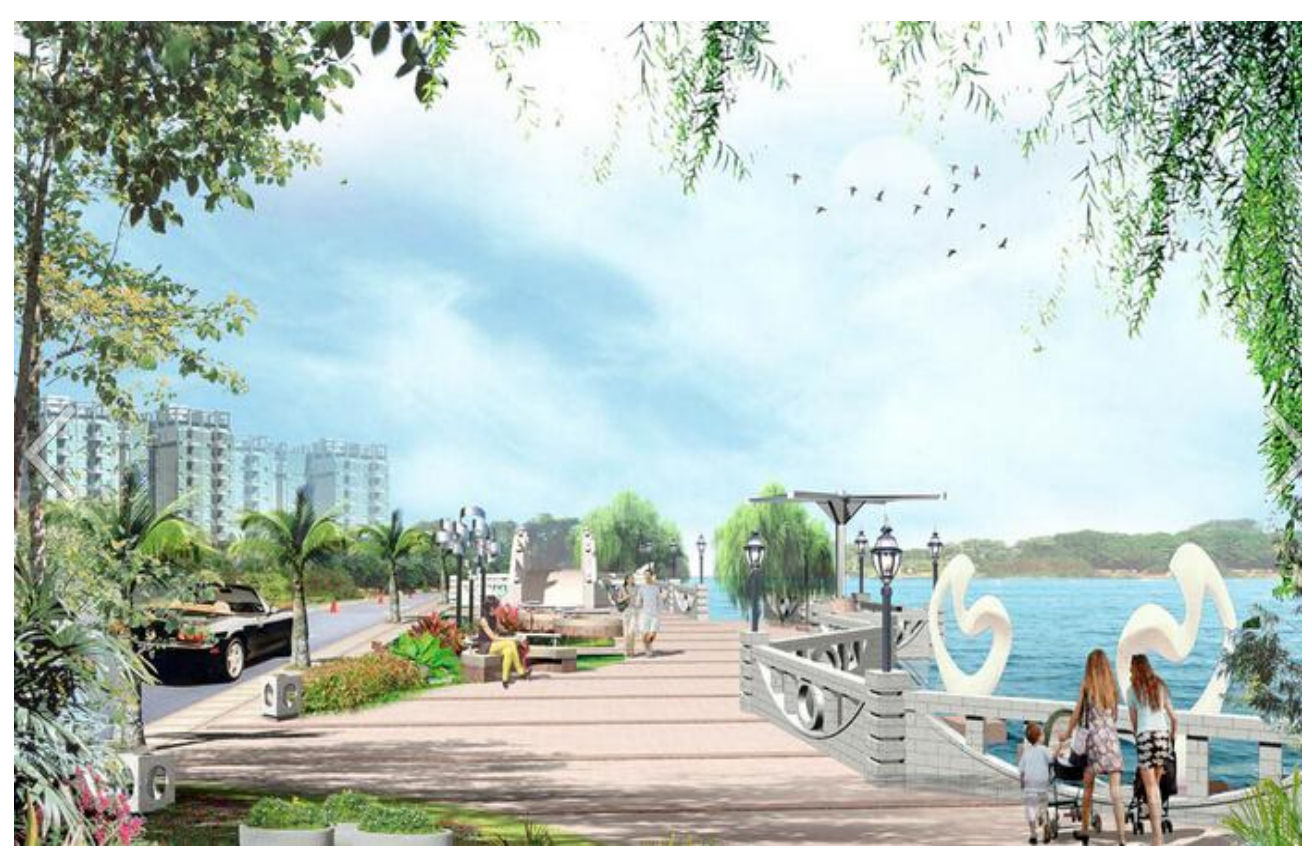

Fig. 2 Schematic Diagram of Landscape Effect in Environmental Artistic Design

(Source: Website)

From Fig. 2, we can clearly see that it is an artistic design drawing of outdoor environment and the picture is a $3 \mathrm{D}$ virtual scene screenshot shown with two-dimensional effect. In fact, it is only a time point of the virtual artistic design. Under the support of virtual reality technique, the environment is in the dynamic state, which is the special effect that not be owned by the traditional environmental artistic design ${ }^{[5]}$.

We can make analysis from an objective point of view that it is understandable that some issues can appear in the process of environmental artistic design by the application of virtual reality technique, which is subject to the characteristics of the technique and makes the environmental artistic design showing a non-realistic aspect. Nevertheless, the virtual reality technique has the strong technique support for the environmental artistic design, which not only can estimate the environmental artistic design alternative, but also can endow the conceptualized color to the environmental artistic design, which can't be achieved in art when the specific items design ${ }^{[6]}$.

(2) Connection of Virtual Reality Technique and Environmental Artistic Design. Under the support of virtual reality technique after the limitations of space or time broken, the environmental artistic design can be used in any specific environment that can be conceived of, the design solutions can be further adjusted with the concept of abstract art and have high artistic value or practical value. Essentially, there exists the professional relevance between the virtual reality technique and the environmental artistic design, and the advantage features of virtual reality technique are more obvious after the virtual reality technique is applied in the field of the environmental artistic design when the creation of the specific environment. Therefore, the integration of the virtual reality technique and the environmental artistic design has more prominent effects ${ }^{[7]}$. From specific point of view, the environmental artistic design is a large engineering design with the integration of 
display, which is to inspire people of all kinds of experiences in the dynamic atmosphere created in the high-tech scene, and then have more intuitive and specific understanding of the concept of environmental artistic design.

\section{(3) Influence of Practical Application of} Virtual Reality Technique on Environmental Artistic Design. It can be found in practice that the profound influence of practical application of the virtual reality technique on the environmental artistic design because the technique application not only can make up for the shortcomings and break through space image and other limitations of traditional environmental artistic design, but also can effectively avoid the potential issues in the actual operation, such as the coordination between people and environment, the design of dynamic environment and other issues. In addition, the cost waste of multiple repetitive designs is avoided under the support of virtual reality technique, and the whole environmental design structure can only be achieved when the virtual reality space is created by the application of computer and multimedia technology. Therefore, the economic and social benefits of physical construction are improved by the application of virtual reality technique to some extent.

\section{Traditional Environmental Artistic Design offset by Virtual Reality Technique. In the} past, the traditional environmental artistic design is subject to the spatial factors. If the specific space scope is excessed in the design solutions, there appears the strong inadaptability and exists the failure in the environmental artistic design, which can be avoided by the application of virtual reality technique. Under the support of the technique, the individual artistic design space created by the environmental artistic design has a certain actual value after the limitations of space or time broken, and makes the audiences on site understanding the unique connotation of the environmental artistic design. The virtual reality system has multi-perceptibility, immersion, reciprocity, conception and other features for the virtual reality technique can lead the operators and the audiences into a realistic simulation environment of outdoor scene. The application of virtual reality technique is a kind of new practical way that people conduct the visualization operation and interaction of the complex date by the computers. Compared to the traditional man-machine interface and the popular windows operation, the virtual reality has the qualitative leap in the technical idea and the actual effects shown are unusual.

\section{Potential Issues of Environmental Artistic Design Alternative effectively avoided by} Application of Virtual Reality Technique. In view of the special technical requirements of environmental artistic design, the multi-disciplinary knowledge hierarchy shall be consolidated to finish the design solutions, and the whole environmental artistic design alternative shall be overturned if there is the careless omission in technique. It shows in practice that the potential issues of environmental artistic design alternative can be avoided by the application of virtual reality technique. Specifically, the three-dimensional modeling technique in the virtual reality system can present the abstract statistical data or concept by table, curve, diagram and other ways. Generally speaking, the virtual reality system can be divided into the foreground simulation 
display and the background technique treatment, and the setup of simulation system model is completed under the support of computer's hardware and software ${ }^{[8]}$. In fact, the virtual reality system itself is a serial of integrated techniques. Each module can exert the maximum utility and some virtual reality of specific situation shall be achieved after the orderly operation of simulation system platform. As a result, previous issues on the design efficiency are avoided by application of the virtual reality technique with the integration of simulation and multimedia technology system. In addition, the asymmetric effect of information of all departments in the process of environmental artistic design can be effectively avoided by the practical application of virtual reality technique. In short, the setup process of environmental artistic design alternative is an integration procedure of the knowledge system of professionals, and the faults in design shall be avoided after the improvement of information exchange if the main points of environmental artistic design are focused on the unified design platform. At present, the integrated operation with science and technology has been achieved in many fields and the efficiency has been improved. In the process of realistic simulation operation, all types of information, geographical position, media reserves and other resources are recorded into the core system based on the situation setup of projection equipment and physical layout model and the integrated application of database system and relevant techniques, in order to timely transfer all the information resources for the systems, which can highlight the application efficiency for the virtual reality technique in the field of environmental artistic design.

The most important function of the virtual reality technique applied in the process of the environmental artistic design is mainly reflected in its interactive aspect, which is different from the implementation of large simple integration display. In the virtual environment of man-machine interaction, the interaction and exchange with the central system can be experienced by partial simulation functions of system, which can show the high-tech interactive integration efficacy of the interactive 3D virtual environmental model. Therefore, some irrational and uncoordinated issues in the environmental artistic design alternative can be reflected by the virtual scene, which favors the technical designer to adjust or improve the design solutions for avoiding the issues appearing in the process of actual environmental construction and the resources waste happening to some extent.

\section{Economic Efficiency of Physical Project Construction improved by Application of} Virtual Reality Technique. From the previous experience, the virtual reality technique has been widely applied in project construction design, environmental artistic design, simulation operation platform and other substantial projects, and has obvious advantages. The main frame construction of 3D simulation model can be built within a very short time by the virtual reality technique under the effect of computer software, which greatly cuts down the period of engineering design in the project field. Meanwhile, if there appears the adjustment of design alternative in the process of design, the physical design contents don't need to be overthrown to rebuild the design objects that done in the traditional environmental design, only the amendment of design adjustment should be conducted in the corresponding design software system, which can reduce the 
cost of engineering design to some extent, avoid the errors in design made by human, improve the quality and efficiency of environmental artistic design, mainly enhance the utilization efficiency of resources in the environmental artistic design projects and improve the economic benefits in the process of physical project operation.

\section{CONCLUSIONS}

The integration of electronic information technology and other modern techniques with the many practical projects can give people new experience, and the realistic virtual reality scenes can bring the excellent multi-sensory feelings to people. The practical application of virtual reality technique in the field of environmental artistic design has made up for the shortcomings in the traditional environmental artistic design, which has effectively avoided the potential issues in actual operation and improved the economic benefits of physical project construction. Therefore, the application of the technique is worth to promote in the relevant industrial projects.

\section{References:}

[1] Liu Yihan, Analysis on Issues of Virtual Reality Technique in Environmental Artistic Design [J]. Modern Decoration (Theory), 08 (08): 90-91 (2013)

[2] Dai Qian, Study on Application of Virtual Reality Technique in Chinese Classical Garden Design [J]. Computer CD Software and Application, 09 (18): 110+112 (2013)

[3] Liu Manzhong, Perfect Performance of Virtual World - Introduction on Application of Virtual Reality Technique in
Environmental Artistic Design [J]. Journal of HuangShi Institute of Technology (Humanities and Social Sciences), 05 (05): 38-39 (2010)

[4] Mi Rui, Study on Application of Virtual Reality Technique in Campus Landscape Design - Taking Campus Landscape Design in Changjiang Professional College for Example [J]. Journal of HuBei Adult Education Institute, 07 (07): 49-51 (2014)

[5] Dai Qian, Huang Xinyuan, Jiang Rui, etc. Survey on Application of Virtual Reality Technique in Garden Planning Design [J]. Electronic Test, 07 (07): 49-51 (2014)

[6] Dai Hongtao, Research on Application of Virtual Reality Technique in Design of Urban Architectural Environment - Taking the Historic Area Planning and Renovation Project in Sinan Roan in Shanghai as example [J]. Journal of Beijing Polytechnic College, 01 (01): 39-41 (2011)

[7] Zhou Xiaocheng, Study on Application of Virtual Reality Technique in Ceramic Product Design based on Man-Machine-Object interaction [J]. Business Culture (The First Half of Month), 06 (06): 379-380 (2011)

[8] An Xing, Li Gang, Xu Linwei, etc. Survey on Application of Virtual Reality Technique in U.S. Military Simulation Training [J]. Electronics Optics \& Control, 10 (10): 45-46 (2011) 